\title{
Dynamic Analysis of Nanomachining with a Multi- Cracked AFM Cantilever
}

Win-Jin Chang, Haw-Long Lee and Yu-Ching Yang

Department of Mechanical Engineering, Kun Shan University, No.195, Kunda Rd., Yongkang Dist., Tainan 71003, Taiwan

The atomic force microscope (AFM) has become an essential tool for imaging the surface topography of conductors and insulators on a micro- and nanoscale level [1]. In addition, AFM has also been powerful in nanomachining [2]. Cracks may occur in the AFM cantilever during the nanomachining experiments or in the fabrication of cantilever. The cantilever with cracks will affect its performance in use. In this article, we analyze the dynamic displacement of a multi-cracked AFM-based nanomachining using the modified couple stress theory [3]. Unlike the classical continuum theory, the modified couple stress theory includes one additional material length scale parameter revealing the micro-scale effect on the response of structures to estimate the size-dependent behaviours. Here the effect of the length scale parameter on the dynamic response of nanomachining with a multi-cracked AFM cantilever is investigated.

Assume an AFM cantilever probe, as shown in Fig. 1, has multiple cracks located at abscissa $\mathrm{C}_{j}$ from the fixed end for $j=1,2,3, \ldots p$, where $p$ is the number of cracks, and in the vicinity of the crack. The rotational spring is used to simulate the crack. The cantilever will be divided into $p+1$ segments by the spring. The spring is assumed to be massless. The probe has the Young's modulus $E$, shear modulus $G$, moment of inertia $I$, density $\rho$, uniform cross-section $A$, and length $L$. When the machining is in progress, the cantilever tip contacts with the specimen and induces a vertical reaction force, $F y(t)$, and a horizontal reaction force, $F_{x}(t)$, which are a function of time $\mathrm{t}$. The cutting system of the AFM cantilever can be modeled as a flexural vibration problem. The governing equation of transverse vibration can be given by [4]

$\left(E I+G A l^{2}\right) \partial^{4} Y_{j} / \partial X^{4}+\rho A \partial^{2} Y_{j} / \partial t^{2}=0, \quad C_{j-1} \leq X \leq C_{j} \quad$ for $j=1,2,3, \ldots p+1$

where $l$ is the material length scale parameter which indicates the size-dependent vibration behavior of the microcantilever based on the modified coupled stress theory and $Y(X, t)$ is the transverse displacement.

For the compatibility of displacement, moment and shear force, the two adjacent portions of the cracked cantilever can be expressed by the following jump conditions as

$$
Y_{j}\left(C_{j}, t\right)=Y_{j+1}\left(C_{j}, t\right), \partial^{2} Y_{j}\left(C_{j}, t\right) / \partial X^{2}=\partial^{2} Y_{j+1}\left(C_{j}, t\right) / \partial X^{2} \text {, and } \partial^{3} Y_{j}\left(C_{j}, t\right) / \partial X^{3}=\partial^{3} Y_{j+1}\left(C_{j}, t\right) / \partial X^{3}
$$

The angular displacement between two segments can be expressed by

$$
W\left(\partial Y_{j+1}\left(C_{j}, t\right) / \partial X-\partial Y_{j}\left(C_{j}, t\right) / \partial X\right)=\left(E I+G A l^{2}\right) \partial^{2} Y_{j}\left(C_{j}, t\right) / \partial X^{2}
$$

where $W$ is the rotational spring constant.

The corresponding boundary conditions for nanomachining by the AFM cantilever are [4,5]

$$
\begin{aligned}
& Y(0, t)=0, \quad \partial Y(0, t) / \partial X=0, \quad\left(E I+G A l^{2}\right) \partial^{2} Y(L, t) / \partial X^{2}=-H F_{x}(t)-M B^{2} \partial^{3} Y(L, t) / \partial X \partial t^{2}, \\
& \left(E I+G A l^{2}\right) \partial^{3} Y(L, t) / \partial X^{3}=F_{y}(t)+M \partial^{2} Y(L, t) / \partial t^{2}
\end{aligned}
$$

where $H$ and $M$ is the height and mass of the tip, respectively. $B$ is the distance between the lower edge of the cantilever and centroid of the tip. The relationship between $F_{x}$ and $F_{y}$ can be expressed as $F_{x}=$ $2 \cot \theta \cdot F_{y} / \pi$ which is obtained from a geometrical relation for a cone shape tip and $\theta$ is a half-conic angle.

The following material properties and geometrical parameters are used [5]: $L=300 \mu \mathrm{m}, E=170 \mathrm{GPa}, \mathrm{G}$ $=66.4 \mathrm{GPa}, I=33.3 \times 10^{-24} \mathrm{~m}^{4}, \mathrm{~A}=10^{-10} \mathrm{~m}^{2}, H=10 \mu \mathrm{m}, \rho=2300 \mathrm{~kg} / \mathrm{m}^{-3}, M=2 \times 10^{-13} \mathrm{~kg}, B=2.5$ 
$\mu \mathrm{m}, P=10^{-8} \mathrm{nN}, \quad F_{y}(\tau)=f_{y}\left(\sin \tau \omega_{\eta} \tau+\sin 3 \tau \omega_{\eta} \tau / 3+\sin 5 \tau \omega_{\eta} \tau / 5\right), r=0.9$ and $f_{y}=0.16 \times 10^{-3}$. The dynamic displacement of the free end of a multi-cracked AFM cantilever can be obtained when Eqs.(1)-(4) are solved and the dimensionless crack flexibility( i.e., $k_{c}=E I / W L$ ) is given. Fig. 2

illustrates the dynamic displacement with three cracks of $C / L=0.3,0.5$, and 0.7 and $k_{c}=0.4$ for various ratios of microcantilever thickness to material length scale parameter $h / l$. It is noted the case of $h / l=\infty$, it implies the result obtained based on the classical beam theory. It can that the displacement increases with increasing the value of $h / l$. This is because the effect of length scale parameter on the displacement becomes small when the value of $h / l$ increa phenomena show size-dependent vibration behavior of the cantilever. For the design of the $\mathrm{c}$ a larger value of $h / l$ should be selected in order to obtain a higher material removal rate.

References:

[1]G. Binnig, C.F. Quate and C. Gerber, Phy. Rev. Lett. 56 (1986) p.930.

[2]J.M. Lee, Y.B. Kim and J.W. Park, J. Nanosci. Nanotech. 13 (2013) p.7741.

[3]F. Yang et al, Int. J. Solids Struct. 39 (2002) p.2731.

[4]M.H. Kahrobaiyan et al, Int. J. Eng. Sci. 48 (2010) p.1985.

[5]T.S. Wu, W.J. Chang and J.C. Hsu, Microelectr. Eng. 71 (2004) p.15.

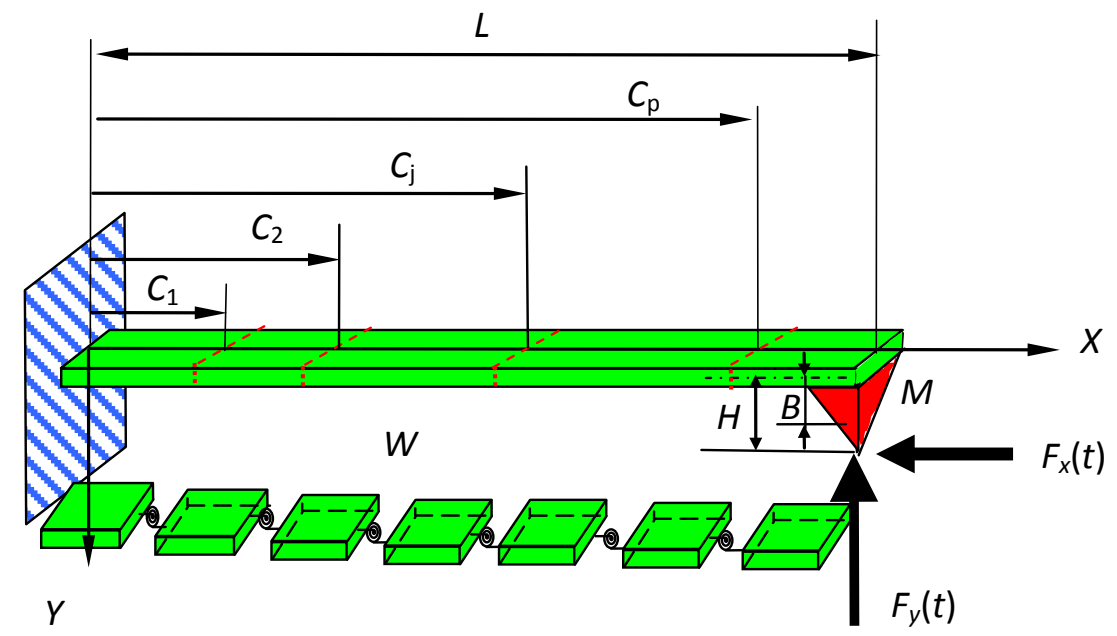

Fig. 1. Schematic diagram of nanomachining on a sample by a multi-cracked AFM cantilever.

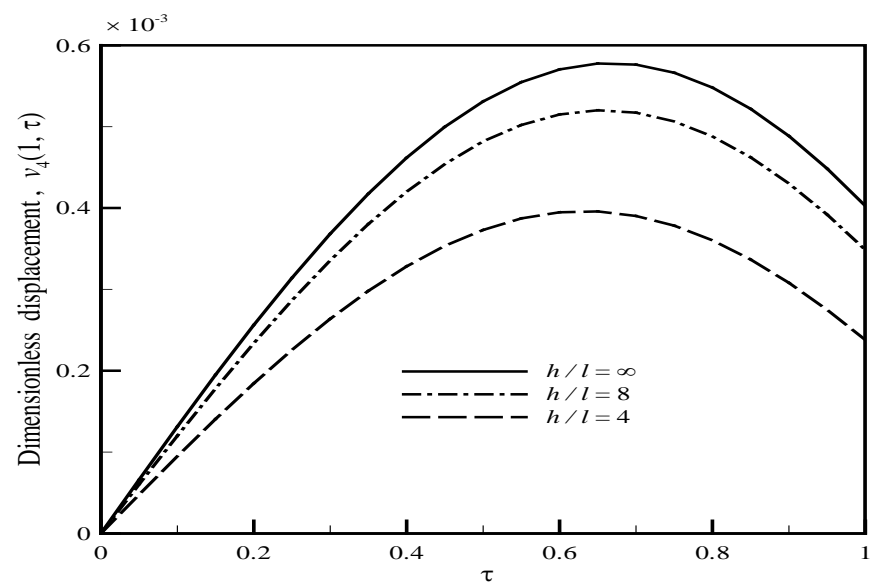

Fig. 2. The dynamic displacement of a three- cracked AFM cantilever with $k_{\mathrm{c}}=0.4$. 